\title{
Kontrollierte Arzneimittelstudien bei Kindern
}

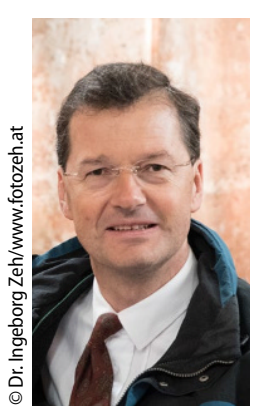

Ao. Univ.-Prof. Dr. Christoph Male
Quelle: Presse-

meldung der

MedUniWien

24.03.2020
_ In der Pro und Kontra- Debatte in Lancet Haematology [1] spricht sich Christoph Male (Foto) von der Universitätsklinik für Kinder- und Jugendheilkunde der MedUni Wien für kontrollierte Arzneimittelstudien bei Kindern aus. Als Beispiel dient ihm die Thrombosebehandlung mit Antikoagulantien. Paul Monagle vom Royal Children's Hospital der University of Melbourne hält dagegen kontrollierte Studien bei Kindern mit Thrombosen für kaum machbar und wenig aussagekräftig. Er plädiert für Dosisfindungsund Beobachtungsstudien.

Male betont, dass randomisiert kontrollierte Studien die verlässlichste Methode seien, um die Wirksamkeit und Sicherheit eines Arzneimittels zu beweisen. Allerdings gebe es insbesondere bei Kindern viele ethische, methodische und praktische Herausforderungen. Wenn ausreichend Daten zu einem Arzneimittel für die Zulassung bei Erwachsenen vorliegen würden, müssten nicht alle Studien gleichermaßen bei Kindern wiederholt werden. Durch systematische Extrapolation der Erwachsenendaten könnten mithilfe moderner statistischer Modelle Voraussagen bezüglich der Dosierungen, Wirksamkeit und auch Sicherheit eines Arzneimittels bei Kindern getroffen werden. Studien bei Kindern seien zwar zur Bestätigung erforderlich, würden aber aufgrund des Vorwissens weniger Kinder benötigen, und in manchen Situationen könne auch auf kontrollierte Studien verzichtet werden. Extrapolation sei allerdings nicht möglich, wenn große Unterschiede in der Erkrankung oder der Arzneimittelwirkung zwischen Erwachsenen und Kindern bestünden. In solchen Fällen seien eigenständige kontrollierte Studien bei Kindern unverzichtbar, um das Nutzen-RisikoVerhältnis einer Therapie für Kinder sicher zu bestimmen, so Male.
Thrombosen treten als Komplikationen bei Kindern mit schweren Grunderkrankungen auf, wie Krebs, Herzfehlern, oder Frühgeburtlichkeit. Kindliche Thrombosen gelten als Beispiel für seltene Erkrankungen, die ein buntes Spektrum von Kindern aller Altersgruppen betreffen. Dementsprechend herausfordernd ist es, Therapiestudien bei diesen Patienten durchzuführen, und lange Zeit gab es hier kaum verlässliche Daten.

Male führt als erfolgreiches Beispiel die kürzlich in Lancet Haematology publizierte kontrollierte Studie bei 500 Kindern mit akuten Thrombosen an, welche die neue orale Substanz Rivaroxaban mit Standard-Antikoagulantien verglich. Es zeigte sich ein Therapieansprechen bei Kindern ähnlich wie bei Erwachsenen. „Daraus lernten wir, dass in dieser Indikation Extrapolation teilweise möglich ist, so dass künftig Kinderstudien kleineren Umfangs genügen", betont Male. Auch zur Antikoagulationsprophylaxe von Thrombosen bei Kindern mit Leukämie und Chemotherapie wurde kürzlich eine kontrollierte Studie mit 950 Kindern erfolgreich abgeschlossen. Wenngleich kontrollierte Studien nicht in allen Indikationen bei Kindern in vollem Ausmaß machbar wären, stellen sie doch das Herzstück der Arzneimittelevaluation dar, denen Beobachtungsstudien des Arzneimitteleinsatzes im Alltag folgen müssen, so Male.

Males Diskussionspartner Paul Monagle anerkennt den Wert von kontrollierten Studien, hält sie jedoch bei Kindern mit Thrombosen für kaum machbar. Seiner Meinung nach seien solche Studien auch nicht geeignet, um für die verschiedenen ThromboseTypen und Altersgruppen verlässliche Aussagen zu treffen. Für die Testung von Antikoagulantien bei Kindern würden Dosisfindungsstudien ausreichen, denen Beobachtungsstudien während des praktischen Einsatzes bei Kindern folgen sollten, welche die klinische Realität besser abbilden würden.

Die Europäische Arzneimittelbehörde (EMA) hat jedenfalls erkannt, dass qualitativ hochwertige Daten für die Zulassung von Arzneistoffen für Kinder erforderlich sind, und verlangt deshalb für jede neue Substanz einen entsprechenden Entwicklungsplan. Für diesen pädiatrischen Entwicklungsplan werden die Firmen von der EMA und deren Experten beraten, zu denen auch Prof. Male gehört.

\section{Korrespondenzadresse}

\section{Christoph Male}

Hämophilie-Ambulanz, Univ.-Klinik für Kinder- und Jugendheilkunde,

MedUniWien

Wien, Österreich

christoph.male@meduniwien.ac.at

\section{Literatur \\ 1. Male C, Monagle P (2020) Are controlled clinical trials of anticoagulation feasible in children? Debate article. Lancet Haema- tology. https://doi.org/10.1016/S2352- 3026(20)30043-0}

Hinweis des Verlags. Der Verlag bleibt in Hinblick auf geografische Zuordnungen und Gebietsbezeichnungen in veröffentlichten Karten und Institutsadressen neutral.

Paediatr. Paedolog. 2020 · 55:156 https://doi.org/10.1007/s00608020-00802-4

(c) Springer-Verlag GmbH Austria, ein Teil von Springer Nature 2020 